\title{
A comparative study of chemotherapy combined with intermittent microwave ablation and chemotherapy in the treatment of oligometastatic non-small cell lung cancer
}

\author{
Linjia Zhu' ${ }^{1}$, Shaofei Yuan' ${ }^{2}$ Meizhen Liang ${ }^{2}$ and Youyi $\mathbf{W u}^{2}$ \\ ${ }^{1}$ Department of Respiratory Medicine, The Third Affiliated Hospital of Wenzhou Medical University, Ruian, China \\ ${ }^{2}$ Department of Oncology, The Third Affiliated Hospital of Wenzhou Medical University, Ruian, China \\ Correspondence to: Youyi Wu, email: xiaomifal@outlook.com \\ Keywords: chemotherapy; intermittent; microwave ablation; oligometastatic; non-small cell lung cancer \\ Received: September 16,2017 Accepted: December 11,2017Ｐublished: January 04, 2018 \\ Copyright: Zhu et al. This is an open-access article distributed under the terms of the Creative Commons Attribution License 3.0 \\ (CC BY 3.0), which permits unrestricted use, distribution, and reproduction in any medium, provided the original author and source \\ are credited.
}

\section{ABSTRACT}

Objective: To compare chemotherapy combined with intermittent microwave ablation and chemotherapy alone in the treatment of oligometastatic non-small cell lung cancer.

Results: In the experimental group $(n=34)$, the total effective rate $(C R+P R)$ was $70.59 \%$ vs. $36.36 \%$ in the control group $(n=33)\left(x^{2}=7.890, P<0.01\right)$. The score of KPS in the experimental group was higher than that in the control group, indicating obvious difference $(P<0.05)$. The median PFS in the control group and the experimental group was (3.6 \pm 0.2$)$ months and (5.4 \pm 0.1$)$ months $\left(x^{2}=42.731\right.$, $P<0.01)$. There was statistical difference that the incidence of pneumothorax and blood sputum in the experimental group was higher than that in the control group $\left(X^{2}=6.031, P<0.05\right)$. However, no significant differences were found regarding other common complications of microwave ablation and chemotherapy.

Conclusions: Chemotherapy combined with intermittent microwave ablation is superior to chemotherapy alone in improving the rate of disease control and the quality of life of patients, as well as prolonging the PFS of patients.

Methods: A total of 34 cases of oligometastatic NSCLC who received chemotherapy with intermittent CT guided microwave ablation and 33 cases of oligometastatic NSCLC patients who received chemotherapy only were enrolled. The efficacy and the adverse effects were evaluated.

\section{INTRODUCTION}

In recent years, the incidence and mortality of lung cancer in the world have shown an obvious upward trend, and have become the leading cause of death of malignant tumors [1]. Surgical treatment is still the first choice for radical treatment of primary bronchogenic carcinoma. However, due to the relatively low diagnostic level for lung cancer in the early phase, the high degree of malignancy of lung cancer and complex biological characteristics of lung cancer and other reasons, most of the patients are diagnosed with lung cancer in the middle and advanced stages, and have lost the chance of surgical treatment [2]. Chemotherapy is the main treatment for advanced lung cancer patients [3]. Nevertheless, the mortality of advanced lung cancer is relatively high because of the side effects and drug resistance [4]. Since the concept of oligometastatic non-small cell lung was raised, the therapeutic strategies for oligometastatic nonsmall cell lung cancer have changed over the last decade from palliative to curative intent [5].

Latterly, the development of minimally invasive tumor ablation therapy in malignant tumors guided by $\mathrm{B}$ ultrasound or CT has attracted more and more attention [6-8]. At present, microwave ablation is widely used in the treatment of liver cancer at home and abroad, which can achieve the effect of killing cancer tissue in situ without surgery [9]. Microwave ablation therapy has its unique 
safety, minimally invasive and effective advantages in the treatment of malignant tumors. Microwave ablation combined with chemical therapy has gradually become one of the important means of clinical treatment of nonsmall cell lung cancer (NSCLC) [10].

According to the National Comprehensive Cancer Network (NCCN) guidelines, oligometastatic NSCLC can be treated locally with surgery or radiotherapy for local control, and combined with systemic chemotherapy [3]. However, there are no related studies of chemotherapy combined with intermittent microwave ablation in the treatment of oligometastatic NSCLC.

\section{RESULTS}

\section{Clinical features}

A total of 67 patients in the control group and the experimental group were enrolled, including 33 cases in the control group, and 34 cases in the experimental group. The treatment program was successfully completed in both groups. Qualitative data including gender, histopathology, and clinical stage were not statistically significant in the two groups at baseline $(P>0.05)$; additionally, no obvious statistical difference was also found regarding quantitative data of age, tumor diameter, and KPS score at baseline $(P>0.05)$. Detailed information was described in Table 1.

\section{KPS scoring improvement}

There was no significant difference in KPS scores between the experimental group and the control group before the microwave ablation $(P>0.05)$. The KPS scores at 1 months and 3 months after operation were significantly different between the experimental group and the control group, indicating statistical difference $(P<0.05)$, which were shown in Table 2 .

\section{Short-term effects}

In the control group $(n=33)$ : the short-term effect was followed up for 6 months, there was 0 patient of CR $(0 \%), 12$ patients of PR $(36.36 \%)$, and the total effective rate $(\mathrm{CR}+\mathrm{PR})$ was $36.36 \%$. In the experimental group $(n=34)$ : the short-term effect was followed up for 6 months, the complete remission (CR) was found in 8 cases $(23.53 \%)$, partial remission (PR) in 16 cases $(47.06 \%)$, and the total effective rate $(\mathrm{CR}+\mathrm{PR})$ was $70.59 \%$. The difference between the two groups was statistically significant $\left(\chi^{2}=7.890, P<0.01\right)$, which was significantly lower in the control group than that in the experimental group.

\section{Progression free survival (PFS)}

The median PFS in the control group and the experimental group was $(3.6 \pm 0.2)$ months and $(5.4 \pm 0.1)$ months, and the difference was significant between groups $\left(\chi^{2}=42.731, P<0.01\right)$. Corresponding median PFS was significantly shorter in the control group than the experimental group. Cumulative PFS curve was shown in Figure 1.

\section{Adverse evens}

There were 5 cases $(17.65 \%)$ of pneumothorax in the experimental group, which was obviously higher than that in the control group $(0 \%)$, with statistical difference $(P<0.05)$. The pulmonary compression accounted for $30 \%$ of the patients with pneumothorax after microwave ablation, and all patients were cured after oxygen inhalation or thoracic puncture and aspiration. The incidence of sputum blood in the experimental group was higher than that in the control group $\left(\chi^{2}=6.031, P<0.05\right)$; however, no evident differences were found regarding other common complications of microwave ablation and chemotherapy, including fever, chest pain, and myelosuppression $(P>0.05)$, which were illustrated in Table 3 .

\section{DISCUSSION}

The quality of life of cancer patients has been paid more and more attention, especially for the elderly patients. The treatment should not merely prolong the patient's survival time, but also consider the quality of life of the patient. In the study, statistical difference was observed regarding the KPS score of the experimental group was higher than that of the control group 1 month after treatment although there was no significant difference in preoperative KPS score between the two groups $(P<0.05)$, indicating that microwave ablation may delay the decrease in quality of life. We believe that microwave ablation can completely kill tumor tissue locally, and some patients can even achieve the equivalent of surgical results, which help to reduce tumor burden of the patient and achieve tumor free survival. In case of avoiding adverse events, it also could improve the quality of life and mental state of the patients by controlling cancer disease.

In the study conducted by Zhigang Wei et al. advanced NSCLC was treated with chemotherapy combined with microwave ablation, and it was estimated that the overall objective effective rate was $74.4 \%$, median PFS was 8.7 months, and overall survival was 21.3 months, which was significantly better than that of the single chemotherapy group [11]. Xu X et al. performed a clinical trial to compare the effect of microwave ablation combined with concurrent chemoradiotherapy and concurrent chemoradiotherapy for locally advanced NSCLC, the results showed that the ratio of effective cases was 85.1 versus $80.4 \%$ for mediastinal lymph node $(P=0.843)$ and 83.0 versus $100 \%$ for pulmonary tumors $(P=0.503)$, respectively, for the RT and ablation groups. Kaplan-Meier analysis demonstrated 2-year OS rate of NSCLC patients in ablation group was higher than RT group, but no statistical difference (log-rank test, 
Table 1: Baseline demographics and clinical characteristics

\begin{tabular}{|c|c|c|c|}
\hline Variable & $\begin{array}{l}\text { Treatment group } \\
\qquad(n=34)\end{array}$ & $\begin{array}{l}\text { Control group } \\
\quad(n=33)\end{array}$ & $P$ value \\
\hline \multicolumn{4}{|l|}{ Patient related factors } \\
\hline Age (years) & $61.27 \pm 10.86$ & $61.88 \pm 9.49$ & 0.807 \\
\hline Gender $(\%)$ & & & 0.729 \\
\hline Male $(n)$ & $24(70.6 \%)$ & $22(66.7 \%)$ & \\
\hline Female $(n)$ & $10(29.4 \%)$ & $11(33.3 \%)$ & \\
\hline KPS $(\%)$ & $81.18 \pm 7.29$ & $79.09 \pm 8.79$ & 0.294 \\
\hline Comorbidities with COPD & & & 0.582 \\
\hline Yes & $13(38.2 \%)$ & $14(42.4 \%)$ & \\
\hline No & $21(61.8 \%)$ & $19(57.6 \%)$ & \\
\hline Ascite/Hydrothorax & 0 & 0 & 0 \\
\hline Pathology Type & & & 0.724 \\
\hline Adenocarcinoma & 14 & 15 & \\
\hline $\mathrm{SCC}$ & 20 & 18 & \\
\hline Tumor size $(\mathrm{cm})$ & $3.8 \pm 1.2$ & $3.9 \pm 1.3$ & 0.851 \\
\hline
\end{tabular}

Table 2: KPS scores in two groups

\begin{tabular}{lccc}
\hline & $\begin{array}{c}\text { Treatment group } \\
(\boldsymbol{n}=\mathbf{3 4})\end{array}$ & $\begin{array}{c}\text { Control group } \\
(\boldsymbol{n}=\mathbf{3 3})\end{array}$ & $\boldsymbol{P}$ value \\
\hline Before ablation & $81.18 \pm 7.29$ & $79.09 \pm 8.79$ & 0.294 \\
1 month later & $81.76 \pm 7.16$ & $72.72 \pm 4.52$ & 0.000 \\
3 months later & $78.82 \pm 6.4$ & $73.03 \pm 4.67$ & 0.000 \\
\hline
\end{tabular}

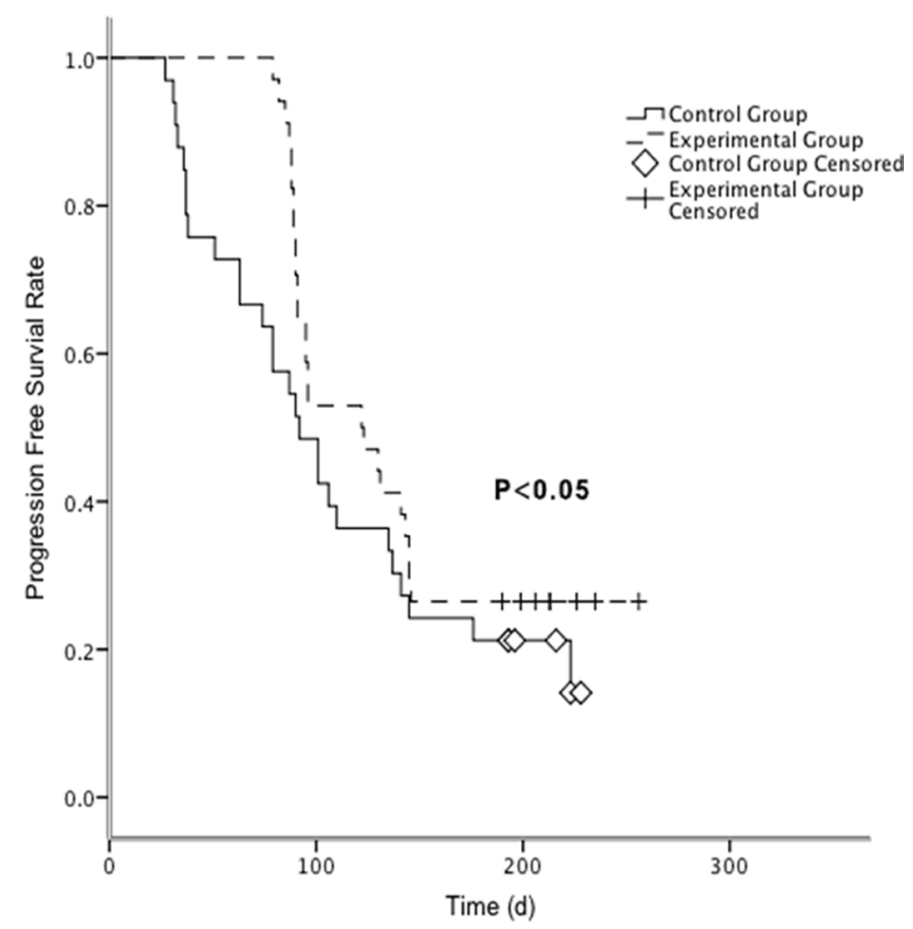

Figure 1: Cumulative PFS curve in the control group and the experimental group. The difference was significant between groups $(P<0.05)$. Median PFS time was significantly shorter in the control group than the experimental group. 
Table 3: Adverse events in two groups

\begin{tabular}{lccc}
\hline Variable $(\boldsymbol{n})$ & $\begin{array}{c}\text { Treatment group } \\
(\boldsymbol{n}=\mathbf{3 4})\end{array}$ & $\begin{array}{c}\text { Control group } \\
(\boldsymbol{n}=\mathbf{3 3})\end{array}$ & $\boldsymbol{P}$ value \\
\hline Aerothorax & $6(17.65 \%)$ & 0 & 0.025 \\
Hemoptysis & $13(38.24 \%)$ & $4(12.12 \%)$ & 0.014 \\
Fever & $2(5.88 \%)$ & $1(3.03 \%)$ & 0.511 \\
Chest Pain & $9(26.47 \%)$ & $7(21.21 \%)$ & 0.614 \\
Myelosuppression & $6(17.65 \%)$ & $8(24.24 \%)$ & 0.507 \\
\hline
\end{tabular}

$P=0.297$ ) [12]. In the present study, the evaluation criteria for clinical effect were divided into two aspects. On the one hand, considering short-term effect, after 6 months of follow-up, the effective rates of the experimental group and the control group were $70.59 \%$ and $36.36 \%$, respectively. The effective rate of the experimental group was higher than that of the control group, and the difference between the two groups was statistically significant $\left(\chi^{2}=7.890\right.$, $P<0.01)$. On the other hand, the median PFS in the control group and the experimental group was $(3.6 \pm 0.2)$ months and $(5.4 \pm 0.1)$ months, respectively, and the difference was significant that the median PFS of the latter group was evidently longer than that of the former group $\left(\chi^{2}=42.731\right.$, $P<0.01)$. Previous have shown that microwave ablation has synergistic effects with systemic chemotherapy [13, 14]. Possible reason may be that chemotherapy can kill G1-S tumor cells, while microwave can kill and inactivate tumor cells in different phases without distinction. Microwave can effectively kill local tumor tissues, systemic chemotherapy can kill tumors of the circulatory system and metastasized tumors exerting the role of systemic treatment. At the same time, microwave can enhance the immune function of the organism and help to make up for the immune suppression caused by chemotherapy. Therefore, it is speculated that microwave ablation therapy has some synergistic effects with chemotherapy.

In the experiment, 34 patients in the experimental group were successfully completed the operation, and no serious complications occurred during the microwave ablation. In the experimental group postoperatively, there were 5 cases $(17.65 \%)$ of pneumothorax in the experimental group, which was obviously higher than that in the control group $(0 \%)$, with statistical difference $(P<0.05)$. The pulmonary compression accounted for $30 \%$ of the patients with pneumothorax after microwave ablation, and all patients were cured after oxygen inhalation or thoracic puncture and aspiration. The incidence of pneumothorax after CT guided percutaneous pulmonary biopsy was estimated to be about $14 ., 4 \%-17.87 \%$, which was similar to that of the incidence of pneumothorax in the study $[15,16]$. Pneumothorax may be induced by microwave puncture for pleural lesions. Secondly, it may be correlated with the relative movement of the visceral pleura and parietal pleura caused by respiratory movements, especially in the course of coughing, which may cause the incision of the puncture needle to the pleura, so as to stimulate pneumothorax [17]. To reduce the incidence of pneumothorax after microwave ablation, we hold that patients should undergo meticulous breath holding training before surgery, and cough treatment should be given. For patients with severe cough. In addition, adequate preoperative anesthesia can help to prevent the patient from severe pain and thus affect the operation. In the study, the coagulation function was normal in both the experimental group and the control group, 13 patients (38.24\%) had bloody sputum in the experimental group, which was significantly higher than that in the control group $(12.12 \%)$, with statistical difference $\left(\chi^{2}=6.031, P<0.05\right)$. The main reason of bloody sputum in the experimental group was the damage of microwave puncture to lung parenchyma. To reduce the incidence of bloody sputum or bleeding after microwave ablation, the enhanced scan should be performed before surgery to determine the distribution and location of the surrounding vessels. Meanwhile, during the preoperative CT scan, the image should be analyzed carefully, combined with the selection of appropriate puncture approach and the avoidance of puncturing the larger vessels. Besides, the frequency of punctures should be reduced as much as possible to achieve success at one puncture. At the end of the puncture operation, repeated CT scans should be performed to observe if there is dynamic bleeding at the site of the puncture. For patients with hemoptysis or bloody symptoms, preoperative hemostasis should be given. At the same time, surgery should be operated by experienced operator who have underwent systematic training, which may in turn help to improve the safety and accuracy of microwave ablation treatment, and to reduce the incidence of pneumothorax, hemothorax and bloody sputum [17].

In the study, the preoperative blood routine and inflammatory indexes were checked in both the experimental group and the control group, indicating no evidence of obvious infection. There was no difference in the incidence of fever between the experimental group and the control group after the microwave ablation treatment $(P>0.05)$. In the experimental group, there were 2 cases of fever after microwave ablation, the temperature was less than $38.5^{\circ} \mathrm{C}$ that was considered to be absorption fever. The temperature was reduced within 
3 days without antibiotic treatment. As for the incidence of myelosuppression, there were 6 cases $(17.65 \%)$ in the experimental group and 8 cases in the control group $(24.24 \%)$. There was no significant difference in incidence between groups $(P>0.05)$, suggesting that microwave ablation may not cause myelosuppression. In conclusion, microwave ablation combined with chemotherapy is an effective treatment for advanced NSCLC. Ablation of the lesion during chemotherapy is more advantageous in improving PFS than that after chemotherapy without significant adverse effects.

However, the sample size of this study is small, and the observation time is short due to the limitation of time, the accuracy of the conclusion and its long-term effect, such as the overall survival rate of patients, should be further studied.

\section{MATERIALS AND METHODS}

\section{Study population}

Patients with advanced NSCLC confirmed by clinical, imaging and pathological examinations who were able to tolerate interventional therapy and chemotherapy were enrolled in the Affiliated Hospital of Wenzhou Medical University from September 2012 to August 2013. The main symptoms were chest pain, hemoptysis, cough, expectoration, fatigue, anorexia, weight loss, etc.. All patients voluntarily signed informed consent and volunteered to participate in this study. All the patients were randomly divided into the control group and the experimental group. Microwave ablation with concurrent chemotherapy was adopted in patients of the control group, and patients in the experimental group received microwave ablation after chemotherapy.

All procedures performed in studies involving human participants were in accordance with the ethical standards of the institutional and/or national research committee and with the 1964 Helsinki declaration and its later amendments or comparable ethical standards.

\section{Inclusion criteria}

1. Pathological diagnosis of lung cancer patients, also including patients with initial treatment; 2 . Pulmonary solitary lesions with observable evaluation; with wildtype EGFR/ALK/ROS1; 3. Stage IV NSCLC with hepatic solitary metastasis only; 4. Patients without radiotherapy, chemotherapy, or molecular targeted therapy 3 weeks before treatment; 5. Normal function of heart and lung; 6. 18-70 years old regardless of gender; 7 . The survival time was expected to be over 3 months; 8 . Good physical condition, Karnofsky performance scale (KPS) score $>70$ points; 9 . Patients with no serious diabetes or coagulopathy; 10 . Routine examination showed no interventional treatment and chemotherapy contraindication; 11. Informed consent was signed by the patient and/or the family.

\section{Exclusion criteria}

1. Patients with pathologically diagnosed small cell lung cancer; 2. Patients with stage I-IIIA by TNM stage who were able to undergo surgical resection; 3 . Patients who had been treated with chemotherapy or radiotherapy or surgery; 4 . Senile and weak patients who were not expected to tolerate interventional therapy and chemotherapy and were expected to survive for $<6$ months; 5 . KPS score $<60$ points; 6 . Patients with heart, liver, kidney and other serious injuries, or combined with diabetes and coagulopathy; 7. Pregnant women, lactating women and all unmarried young patients.

\section{Therapeutic method}

\section{Treatment plan}

All the patients were randomly divided into the control group and the experimental group.

Control group: 6 cycles of chemotherapy alone.

Experimental group: CT guided microwave ablation for hepatic lesions after 2 cycles of chemotherapy, then, 2 cycles of chemotherapy were performed and CT guided microwave ablation was used to treat the pulmonary lesions, followed by another 2 cycles of chemotherapy.

\section{Chemotherapy protocol}

1. The GP protocol was used in NSCLC patients with squamous cell carcinoma confirmed histologically: Gemcitabine, $1000 \mathrm{mg} / \mathrm{m}^{2} \mathrm{~d} 1$, d8; Cisplatin, $75 \mathrm{mg} / \mathrm{m}^{2}$ (total volume) d1-3; or DP protocol: Docetaxel, $75 \mathrm{mg} / \mathrm{m}^{2}$ d1; Cisplatin, $75 \mathrm{mg} / \mathrm{m}^{2}$ (total volume) $\mathrm{d} 1-3$; 21 days for 1 period, in a total of 6 cycles.

2. The AP protocol was used in NSCLC patients with adenocarcinoma confirmed histologically: Pemetrexed, 500 $\mathrm{mg} / \mathrm{m}^{2} \mathrm{~d} 1$; Cisplatin, $75 \mathrm{mg} / \mathrm{m}^{2}$ (total volume) $\mathrm{d} 1-3$; or DP protocol: Docetaxel, $75 \mathrm{mg} / \mathrm{m} 2 \mathrm{dl}$; Cisplatin, $75 \mathrm{mg} / \mathrm{m}^{2}$ (total volume) d1-3; 21 days for 1 period, in a total of 6 cycles.

\section{Microwave ablation}

\section{Instrument and equipment}

(1) 16-slice spiral CT (Siemens, Germany); (2) Cool-tip microwave therapeutic apparatus (Fuzhong medical high-tech Co., Ltd); (3) Cool-tip microwave knife (Fuzhong medical high-tech Co., Ltd);

\section{Preoperative preparation}

(1) Improvement of the heart and lung function, lung function could be tested in patients with suitable condition. Patients were given sedative and cough medicine if the patients had symptom of cough, and hemostatic treatment was adopted if there was hemoptysis and bloody symptoms in the sputum; (2) Patient or the 
family member signed the informed consent prior to the operation as well as the informed consent for cool-tip microwave tumor ablation; (3) All patients were subjected to routine laboratory tests including routine blood test, blood coagulation time, liver and kidney function, blood sugar and biochemical tests, six serum hepatitis B markers and HIV tests before treatment;

\section{Ablation procedure}

The CT scan was performed firstly, and the puncture point, the angle and the depth of the needle were determined according to the scanning results. The approach of percutaneous access was determined by tumor size, morphology, location, adjacent structures, and process pathways. After the local anesthesia with $5 \mathrm{ml} \mathrm{2 \%}$ lidocaine, the microwave needle was inserted according to the designed angle and depth. Then, the CT scan was repeated to confirm the point of the needle to its intended position, and the long axis of the tumor was tried to be prolonged, lately, the microwave needle was inserted to the far side of the tumor. Following the verification of the position of the needle tip, the puncture needle was fixed near the skin, and the physiological saline inlet pipe and the microwave tube were connected, subsequently, the water circulation system was opened, followed by the selection of the power according to the size of the lesion, the surrounding tissue and organ condition, 40-80 $\mathrm{W}$ generally, and the treatment time was 12-20 min on the basis of the power size and local blood supply of the lesion. At the end of the treatment, the patient was instructed to hold breath, the needle was pulled out in the course of treatment to prevent bleeding and needle track implantation. After therapy, CT scanning was performed to observe the changes of lesions and the occurrence of complications such as pneumothorax and hemothorax. The patients were instructed to stay in bed, in combination with the treatment of anti-infection, hemostasis, relieving cough and relieving pain. Intensive monitoring was performed to see if there were any surgical complications or other complications (Figures 2, 3).

\section{Postoperative treatment}

(1) Patients were transported back to the ward using oxygen bags and nasal catheters for oxygen inhalation, and were escorted by special medical staff.

(2) The patient was sent to the ICU for 4 hours and then transferred to the general ward when the condition was stable.

(3) Chest radiographs were taken for patients $24 \mathrm{~h}$ or $48 \mathrm{~h}$ after surgery, to observe the occurrence of secondary pneumothorax and hemothorax.

(4) All the patients were given hemostatic drugs to prevent bleeding. The usage of antibiotics was determined according to the situation after operation to prevent infection.

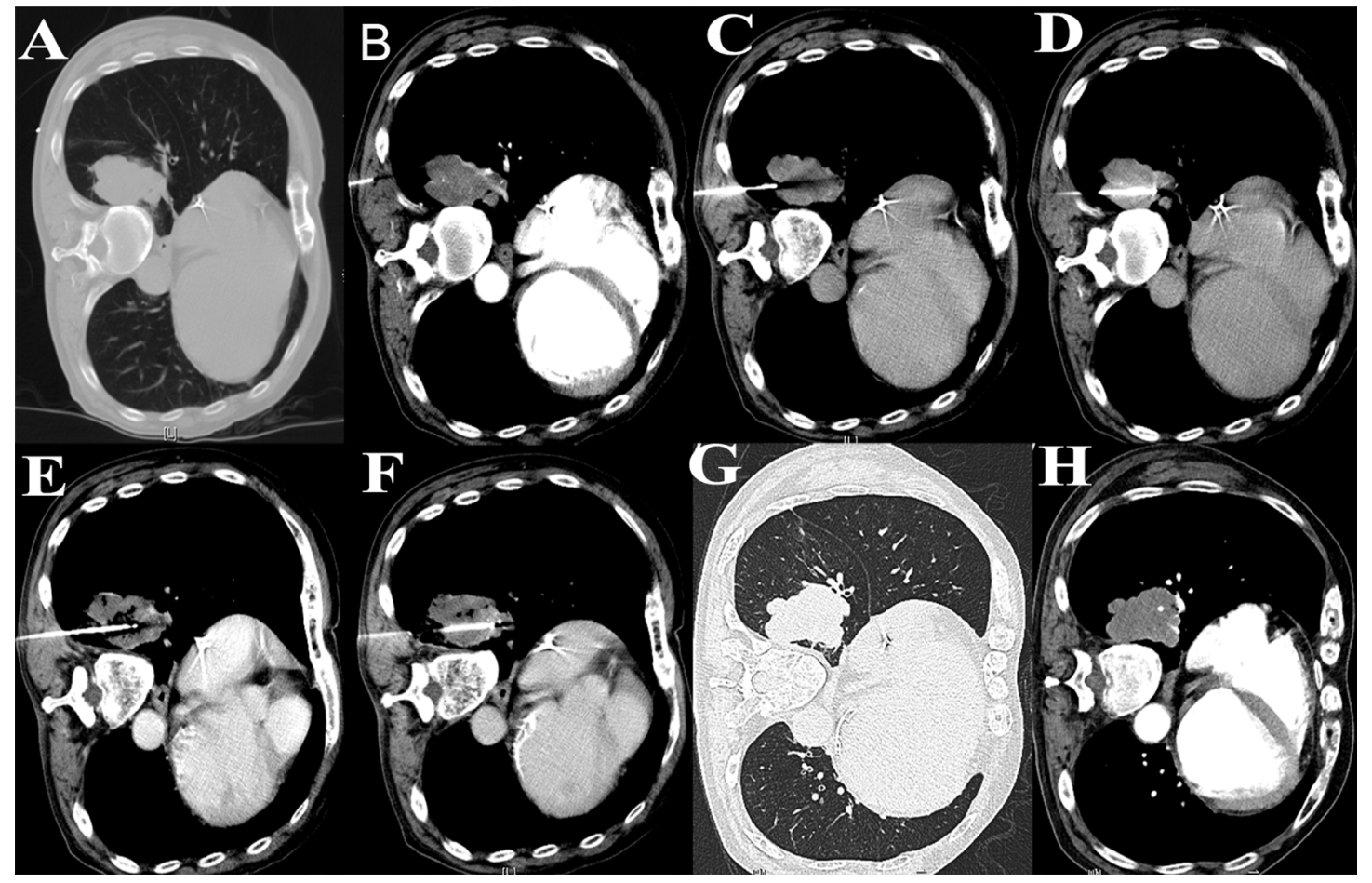

Figure 2: Microwave ablation procedure in primary cancer in lung. Plain CT scan was performed before puncture procedure to map the tumor size and location (A). For large mass, contrast-enhanced CT was to clearly show vessels in the tumor to avoid bleeding caused by puncture damage (B). The step-by-step puncture procedure was shown in $(\mathbf{C}, \mathbf{D})$. Repeated ablation was performed to ensure complete ablation $(\mathbf{E}, \mathbf{F})$. Plain CT scan was performed after ablation procedure to exclude aerothorax and bleeding $(\mathbf{G}, \mathbf{H})$. 


\section{Evaluation criterion}

Two groups of patients were followed up every 2 cycles after chemotherapy. Chest CT was conducted 1 month after microwave ablation, and the whole-abdominal and chest $\mathrm{CT}$ were reviewed at 1,3 , and 6 months after treatment. Simultaneously, CT enhanced scan was used to evaluate the activity of the lesions, and the efficacy was evaluated objectively according to the modified Response Evaluation Criteria in Solid Tumors (mRICIST) [10].

\section{Follow-up}

All patients who were followed up for $\mathrm{CR} / \mathrm{PR} / \mathrm{SD}$ were treated with the original regimen. The effective rate was $(\mathrm{CR}+\mathrm{PR}) / 100 * 100 \%$. For PD and died patients, follow-up was terminated and PD patients received additional treatment. The PFS of the two groups was counted and the adverse reactions were observed in both groups at the same time.

\section{Statistical analysis}

The data were analyzed by statistical software SPSS 16.0. Measurement data were compared using $t$-test, and the $\chi^{2}$ test was used in the comparison of enumeration data. Kaplan-Meier method and Log Rank method were applied for comparison PFS between groups. The difference was statistically significant when $P<0.05$.

\section{Author contributions}

Linjia Zhu wrote the manuscript; Shaofei Yuan analyzed the results. Meizhen Liang prepared the figure and tables. Youyi $\mathrm{Wu}$ raised the idea and guided the whole paper.

\section{ACKNOWLEDGMENTS}

We expressed our thanks to the department of imaging stuffs.

\section{CONFLICTS OF INTEREST}

All authors declare no conflicts of interest.

\section{FUNDING}

There was no funding.

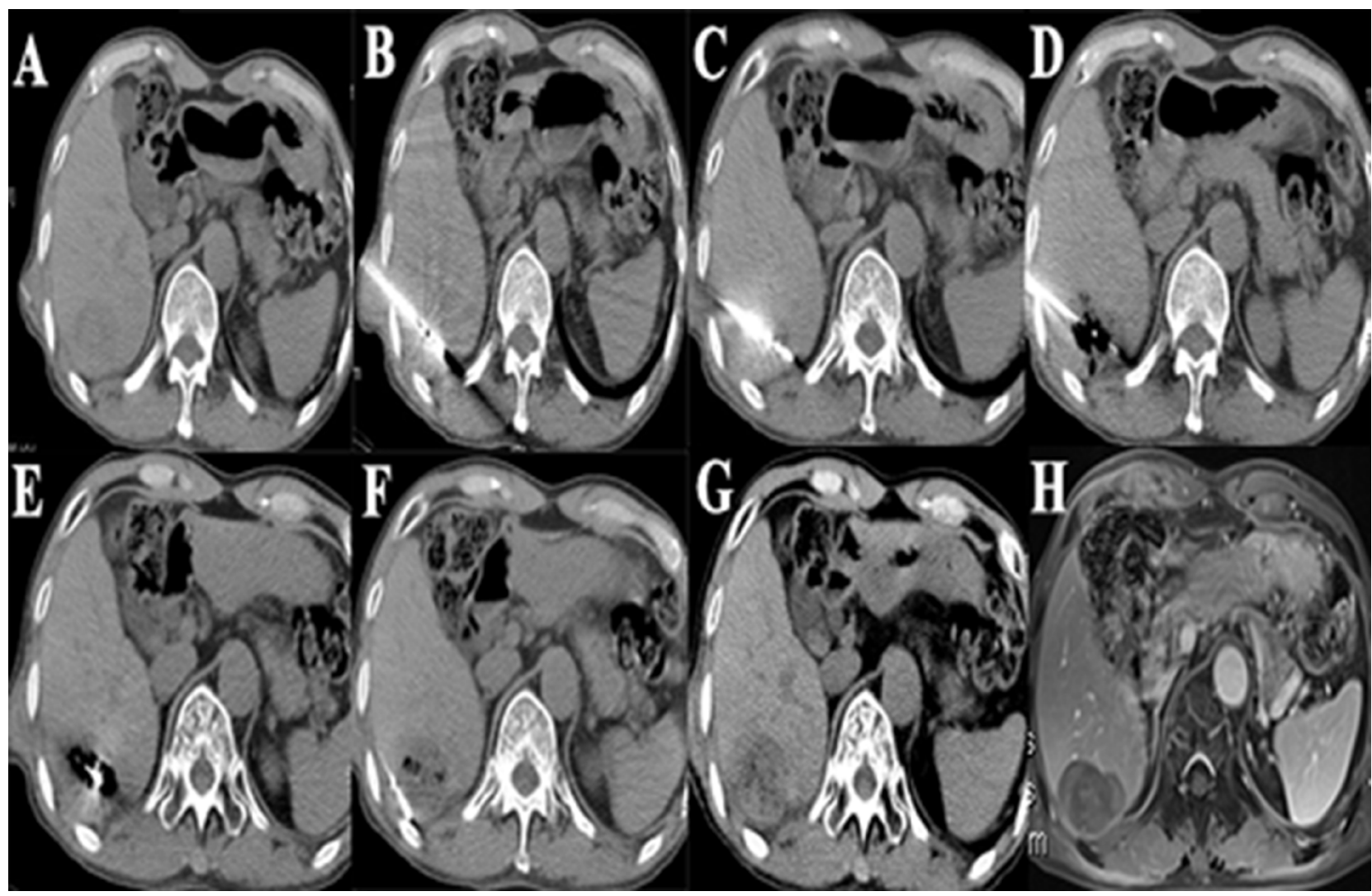

Figure 3: Microwave ablation procedure in metastatic cancer in liver. Plain CT scan was performed before puncture procedure to map the tumor size and location (A). The step-by-step puncture procedure was shown in (B, $\mathbf{C})$. Repeated ablation was performed to ensure complete ablation (D-F). Plain CT scan was performed after ablation procedure to exclude bleeding (G). MRI was performed in follow-up time to observe the recurrence in ablation site $(\mathbf{H})$. 


\section{REFERENCES}

1. Torre LA, Bray F, Siegel RL, Ferlay J, Lortet-Tieulent J, Jemal A. Global cancer statistics, 2012. CA Cancer J Clin. 2015; 65:87-108. https://doi.org/10.3322/caac.21262.

2. Jorge SE, Kobayashi SS, Costa DB. Epidermal growth factor receptor (EGFR) mutations in lung cancer: preclinical and clinical data. Braz J Med Biol Res. 2014; 47:929-39.

3. Ettinger DS, Wood DE, Akerley W, Bazhenova LA, Borghaei H, Camidge DR, Cheney RT, Chirieac LR, D'Amico TA, Dilling TJ, Dobelbower MC, Govindan R, Hennon M, et al. NCCN Guidelines Insights: NonSmall Cell Lung Cancer, Version 4.2016. J Natl Compr Canc Netw. 2016; 14:255-64. https://doi.org/10.6004/ jncen.2016.0031.

4. Scagliotti GV, Parikh P, von Pawel J, Biesma B, Vansteenkiste J, Manegold C, Serwatowski P, Gatzemeier U, Digumarti R, Zukin M, Lee JS, Mellemgaard A, Park K, et al. Phase III study comparing cisplatin plus gemcitabine with cisplatin plus pemetrexed in chemotherapy-naive patients with advanced-stage non-small-cell lung cancer. J Clin Oncol. 2008; 26:3543-51. https://doi.org/10.1200/JCO.2007.15.0375.

5. North DA, Groeschl RT, Sindram D, Martinie JB, Iannitti DA, Bloomston M, Schmidt C, Rilling WS, Gamblin TC, Martin RC. Microwave ablation for hepatic malignancies: a call for standard reporting and outcomes. Am J Surg. 2014; 208:284-94. https://doi.org/10.1016/j.amjsurg.2014.02.002.

6. Floridi C, De Bernardi I, Fontana F, Muollo A, Ierardi AM, Agostini A, Fonio P, Squillaci E, Brunese L, Fugazzola C, Carrafiello G. Microwave ablation of renal tumors: state of the art and development trends. Radiol Med. 2014; 119:533-40. https://doi.org/10.1007/s11547-014-0426-8.

7. Lei F, Jing Z, Bo W, Dongmei H, Zhencai L, Xue J, Fang W, Hongyu Z, Jintao R. Uterine myomas treated with microwave ablation: the agreement between ablation volumes obtained from contrast-enhanced sonography and enhanced MRI. Int J Hyperthermia. 2014; 30:11-8. https:// doi.org/10.3109/02656736.2013.853107.

8. Wang ZL, Liang P, Dong BW, Yu XL, Yu DJ. Prognostic factors and recurrence of small hepatocellular carcinoma after hepatic resection or microwave ablation: a retrospective study. J Gastrointest Surg. 2008; 12:327-37. https://doi.org/10.1007/s11605-007-0310-0.

9. Wei Z, Ye X, Yang X, Zheng A, Huang G, Li W, Ni X, Wang J, Han X. Microwave ablation in combination with chemotherapy for the treatment of advanced non-small cell lung cancer. Cardiovasc Intervent Radiol. 2015; 38:135-42. https://doi.org/10.1007/s00270-014-0895-0.
10. Ronot M, Bouattour M, Wassermann J, Bruno O, Dreyer C, Larroque B, Castera L, Vilgrain V, Belghiti J, Raymond E, Faivre S. Alternative Response Criteria (Choi, European association for the study of the liver, and modified Response Evaluation Criteria in Solid Tumors [RECIST]) Versus RECIST 1.1 in patients with advanced hepatocellular carcinoma treated with sorafenib. Oncologist. 2014; 19:394402. https://doi.org/10.1634/theoncologist.2013-0114.

11. Xu X, Ye X, Liu G, Zhang T. Targeted percutaneous microwave ablation at the pulmonary lesion combined with mediastinal radiotherapy with or without concurrent chemotherapy in locally advanced non-small cell lung cancer evaluation in a randomized comparison study. Med Oncol. 2015; 32:227. https://doi.org/10.1007/s12032-015-0672-1.

12. Luo J, Wu X, Zhou F, Zhou Y, Huang T, Liu F, Han G, Chen L, Bai W, Wu X, Sun J, Yang X. Radiofrequency hyperthermia promotes the therapeutic effects on chemotherapeutic-resistant breast cancer when combined with heat shock protein promoter-controlled HSV-TK gene therapy: Toward imaging-guided interventional gene therapy. Oncotarget. 2016; 7:65042-51. https://doi. org/10.18632/oncotarget.11346.

13. Tang Y, McGoron AJ. Increasing the rate of heating: a potential therapeutic approach for achieving synergistic tumour killing in combined hyperthermia and chemotherapy. Int J Hyperthermia. 2013; 29:145-55. https://doi.org/10.3109/02656736.2012.760757.

14. Guimaraes MD, Andrade MQ, Fonte AC, Benevides G, Chojniak R, Gross JL. Predictive complication factors for CT-guided fine needle aspiration biopsy of pulmonary lesions. Clinics (Sao Paulo). 2010; 65:847-50.

15. Laspas F, Roussakis A, Efthimiadou R, Papaioannou D, Papadopoulos S, Andreou J. Percutaneous CT-guided fine-needle aspiration of pulmonary lesions: Results and complications in 409 patients. J Med Imaging Radiat Oncol. 2008; 52:458-62. https://doi.org/10.1111/j.1440-1673. 2008.01990.x.

16. Yildirim E, Kirbas I, Harman A, Ozyer U, Tore HG, Aytekin C, Boyvat F. CT-guided cutting needle lung biopsy using modified coaxial technique: factors effecting risk of complications. Eur J Radiol. 2009; 70:57-60. https://doi. org/10.1016/j.ejrad.2008.01.006.

17. Sun YH, Song PY, Guo Y, Sheng LJ. Computed tomography-guided percutaneous microwave ablation therapy for lung cancer. Genet Mol Res. 2015; 14:4858-64. https://doi.org/10.4238/2015.May.11.18. 